\title{
Différentes facettes de la mixité des discours rapportés
}

\author{
Elżbieta Biardzka \\ Université de Wrocław (Pologne) \\ ebiardzka@wp.pl
}

\section{Préliminaires}

Les emplois des expressions guillemetées ont fait l'objet de nombre de travaux. Cependant, leur quantité ne semble pas aller de pair avec la diversité des points de vue qu'ils adaptent. Tout au contraire, leur thématique est focalisée surtout sur la problématique du discours dit indirect (désormais DI) et sur le critère typographique. Rappelons que déjà en 1929, Mikhaïl Bakhtine (1978) évoque les emplois verbalo-analytiques du DI. A ses yeux, ils réunissent le plan analytique concentré sur le contenu des paroles rapportées et le plan d'expression. En 1978, Jacqueline Authier-Revuz décrit encore une fois le discours indirect «quasi-textuel » $(1978: 28$ et 73-74) dont les segments reformulés contiennent aussi des «îlots textuels ». Pour elle, cette forme du discours rapporté (désormais DR) fonctionne sur le mode général sémiotiquement complexe qui consiste à « utiliser des mots des autres » dans sa propre parole, c'est-à-dire à jouer sur la modalisation autonymique (1978 : 73). Dans sa thèse consacrée aux verbes de communication, Marie Madeleine de Gaulmyn (1983) propose la distinction entre ce qu'elle appelle des « formes simples » et des «formes mixtes» du discours rapporté ${ }^{2}$ et le discours indirect libre (1983 : 231). Les formes mixtes comprennent dans la classification de Gaulmyn sept cas et sont délimitées suivant le critère formel, ou, plus précisément, typographique que sont les guillemets ${ }^{3}$. Autrement dit, Gaulmyn conçoit les formes mixtes du DR somme une sorte d'hybridation des formes simples et des expressions guillemetées, îlots textuels, d'après elle quasiment « empruntées » au DD. La dénomination «mixte » est alors reprise par beaucoup de chercheurs. Certains parmi eux, tels Rosier (1999 : 201-246) ou Tuomarlà $(2000: 32,39-40)$ soutiennent que les fragments guillemetés à l'intérieur de l'énoncé rapporté relèvent simplement du DD et se trouvent insérés en DI. Les formes « mixtes » réunissent ainsi et les caractéristiques du DD et celles du DI aussi bien du point de vue typographique et énonciatif que sémiotique.

\section{Retour aux données empiriques}

Tous ces travaux, tout en traitant des expressions guillemetées à l'intérieur des séquences du DR s'attachent à analyser surtout l'un de ses segments constitutifs, à savoir le segment qui représente les paroles citées et qui, pour utiliser l'expression de Gérard Genette, «verbalise du verbal » (1972: 186203). Cependant, selon l'approche assez communément admise actuellement ${ }^{4}$ et promue entre autres par Jacqueline Authier-Revuz (1992, 1993), Dominique Maingueneau (1987, 1994) et Laurence Rosier (1999: 126-129), le discours rapporté comprend deux segments: celui qui relate les données de l'énonciation primaire (les données situationnelles) et celui qui figure le dit de quelqu'un ${ }^{5}$. Pris nécessairement ensemble, la citation au sens large, aussi bien littérale que reformulée (désormais Cit) et le discours citant qui l'accompagne (désormais DC) constituent, sur le plan textuel, les séquences binaires du DR qui ouvrent l'accès cognitif au plan diégétique, c'est-à-dire qui permettent au lecteur de (se) former des représentations des actes de discours/langagiers réels. De la sorte, le DR peut se figurer sous l'équation suivante : $\mathrm{DR}=\mathrm{DC}+\mathrm{Cit}^{6}$. Dans cet ordre d'idées, il nous parait judicieux de considérer que les îlots textuels peuvent apparaitre dans les deux segments du DR, c'est-à-dire aussi bien dans le segment Cit que dans le segment $\mathrm{DC}^{7}$. Quant à l'étude de ce dernier, commentaire dont le rapporteur accompagne la Citation, ses analyses sont plutôt rares dans les travaux et dans les manuels. À 
l'exception du verbum dicendi qui a toujours éveillé un certain intérêt, ce terrain semble être nettement en friche.

\subsection{Discours citant avec îlot textuel}

Le statut discursif du DC est fort complexe ${ }^{8}$. Il fait partie du DR mais il ne relève pas pour autant de la Citation. Sans faire partie du reste du texte «muet» (qui raconte des événements et non les paroles), il partage avec lui la propriété référentielle de verbaliser du non-verbal. Ainsi, l'intervention de l'îlot dans le domaine énonciatif du DC entraîne des effets inattendus. Le DC contient alors du "verbal verbalisé » qui, à côté du non-verbal verbalisé, fonctionne comme commentaire de la Cit. En voici des exemples illustrant le DC guillemeté :

(1) «Je lis des phrases un peu tous les jours et je les mets dans ma tête », explique ce jeune homme de vingt ans, qui fait des allers-retours entre « le bled » en Algérie et les Minguettes, à Vénissieux, où vit son père. (Le Monde, mercredi, le 12 février $2003 / 10)^{9}$

(2) «Nous sommes proches de la fin d'une longue route. Nous avons assayé la diplomatie, les sanctions économiques et les embargos, les incitations positives comme le programme "Pétrole contre nourriture », les inspections sur le terrain, les frappes aériennes limitées. Toutes ces approches ont échoué et toutes les options sont épuisés ». Sous-entendu, hormis la guerre. Ainsi parlait, le 20 janvier, à Washington, le secrétaire américain de la défense Donald Rumsfeld, face aux dirigeants de l'Association des officiers de réserve dans les trois armées, à propos du «cas unique » que représente le désarmement de l'Irak pour les Etats Unis. (Le Monde, dimanche, 26/lundi, 27 janvier 03/2)

(3) Rien, selon Tony Blair, ne serait pire que "l'inaction»: "Ce ne serait pas le chemin de la paix, mais celui de la folie et de la faiblesse, qui conduirait à un conflit plus sanglant, plus incertain, et plus dévastateur. Laisser Saddam en place ne serait en rien synonyme de paix ㅃ‥ (Le Monde, jeudi 27 février 03/2)

(4) A des collaborateurs qui le mettaient en garde contre «les dommages collatéraux diplomatiques », notamment dans ses relations avec les Etats Unis, le chancelier avait répondu en substance que, pour ne pas avoir de problèmes, il suffisait de perdre les élections. (Le Monde, mercredi, 15 janvier $03 / 2$ )

Les îlots textuels (soulignés) qui apparaissent en (1-2) peuvent être interprétés comme exposants d'une non-cö̈ncidence $d u$ discours à lui-même : dans le $\mathrm{DC}$, le journaliste indique un emprunt au discours extérieur par rapport à son propre discours verbalisant les données de la situation primaire ( $c f$. AuthierRevuz 1988, 1995, 1997 :35). L'expression de la non-coïncidence du dire n'est accompagnée d'aucune glose, ce sont les guillemets seuls qui véhiculent ce « qui ne va pas de soi ». La prise en charge de l'îlot n'est pas toujours univoque et automatique mais fort interprétative : par exemple, le mot «bled» ne provient pas nécessairement du parler du « jeune homme de vingt ans » (même si cette interprétation est possible) mais peut être par exemple emprunté à la communauté dont il fait partie. A notre avis, dans ce type d'emploi, le DC a un caractère plutôt complexe et non pas mixte: il cumule le rapport d'événements non verbaux (données situationnelles de l'acte de parole rapporté) et le rapport de paroles, c'est-à-dire il fait le cas de la modalisation autonymique ${ }^{10}$ que Marc Wilmet (1998: 566-567) aurait appelée discours absorbé. Ainsi, sur le mode référentiellement complexe, du non verbal se mêle au verbal. Les exemples (3-4) représentent des cas de cumul encore plus poussés. En (3), le DC complexe se figure par la séquence : Cit mixte + «selon A »+Cit mixte. Selon la configuration : DR $=$ Did + Cit on aura DR $=$ Cit mixte + «selon A» + Cit mixte + Cit reproduction intégrale. L'attribution du dire exprimée par 
« selon A » semble univoque : il s'agit certainement du propos cité de Tony Blair. De la sorte, dire que les DC verbalisent uniquement du non-verbal, alors que seule la Cit verbaliserait du verbal, revient à présenter une vision trop simpliste de la pratique du DR.

\subsection{Citation (reformulée) avec îlot textuel}

La mixité est d'habitude décrite comme intrusion d'îlots textuels dans un segment reformulé (cf. à ce propos Authier-Revuz 1978: 73-74, Rosier 1999: 233-237). Les îlots textuels incorporés dans le segment Cit reformulé mettent en jeu uniquement du verbal: deux sortes de rapport de paroles se combinent : la reformulation s'associe simplement à la reproduction (segments soulignés):

(5) Mgr Laghi a déclaré que l'entretien avec Bush avait été «bon». (Le Monde, vendredi, 7 mars $03 / 2$ )

(6) Le général Myers a assuré que des efforts «extraordinaires » seront faits pour épargner les civils, mais il n'a pas caché que des "gens vont mourir». (Le Monde, vendredi, 14 février $03 / 2$ )

A ce qu'il nous semble, il serait peut-être utile de réserver la dénomination de mixte aux caractéristiques typographiques et sémantico-énonciatives des segments Citations et non pas aux discours rapportés entiers. Ainsi, la Cit est discursivement mixte car elle cumule deux types opposés de représentation du discours autre : la reformulation et la reproduction, et le fragment guillemeté est automatiquement mis à la charge de l'énonciateur cité. Une telle conception de la mixité permet de délimiter et de nommer les segments citationnels et de les opposer aux reproductions et aux reformulations. Ainsi, les discours rapportés contiendraient trois types de citations : reproduites, reformulées et mixtes. Elles peuvent se combiner ensuite avec différents types énonciatifs et grammaticaux de DC. En discours, le montage de deux segments donne un nombre important d'occurrences ${ }^{11}$. Une telle approche paraît d'autant plus pertinente que ce sont précisément les questions de combinatoire des segments Cit guillemetés et des segments DC qui sont le plus souvent discutées dans les travaux.

\section{Problématique de la combinatoire DC + Cit}

En général, les relations entre le DC et la Cit se définissent par deux cas de figure. Soit le locuteur rapporte les paroles de différentes personnes sans les assimiler à la syntaxe de son propre énoncé, soit il les intègre dans son discours conformément aux règles de la syntaxe. Dans le premier cas, nous avons affaire à une combinatoire libre, dans le second cas, nous affrontons une combinatoire contrainte ( $c f$. note $\mathrm{n}^{\mathrm{o}} 6$ ). C'est dans le cadre de cette dernière qu'on examine d'habitude l'intervention des îlots textuels dans le DR. Ainsi, si l'idée de la mixité a été souvent critiquée par Authier-Revuz, c'est parce que pour elle, les expressions guillemetées pleinement intégrées au contexte syntaxique environnant ( c'est-àdire dans le DC), avec déictiques ramenés à un seul cadre énonciatif (celui du rapporteur), ne représentent aucune rupture, ni sémiotique, ni syntaxique, ni énonciative. Aussi, Authier-Revuz évite-telle de parler dans ce cas de mixité quelconque. Selon elle, une forme qui est énonciativement homogène ne peut pas être mixte (1993 : 14, 2004 : 47-49). Eventuellement, elle représente un cas de DR complexe. Le parti pris d'Authier-Revuz est fortement défendu dans les travaux récents de Greta Komur (2003a, $2004 \mathrm{a}$ et 2004b). Pour elle aussi, lorsque les îlots textuels s'intègrent par des ajustements déictiques au contexte du DR, on a affaire non pas à des formes mixtes quelconques mais à des variantes complexes du DR. Seulement lorsque l'îlot textuel garde son propre cadre énonciatif sans s'ajuster à celui du contexte qui l'entoure, on a affaire à des formes « hybrides » (ou, éventuellement, « mixtes » ${ }^{2}$ ) ambiguës du point de vue sémiotique. Chez cet auteur, la description du DR est donc particulièrement affinée par une distinction supplémentaire (complexe/hybride, éventuellement mixte) qui se fait à l'appui des critères sémiotiques et énonciatifs. En effet, dans certains cas, on peut parler de véritables DR « hybrides » ${ }^{13}$ 
dont les Cit sont postposées au DC avec «que », juste comme dans la séquence (7), contenant une Cit reproduction dont les déictiques restent intacts :

(7) Interrogé par CNN, l'ancien président Bill Clinton a estimé que «sur le plan militaire, nous pouvons battre Saddam Hussein facilement et rapidement ». (Le Monde, vendredi, 14 février $03 / 2$ )

En (7), les déictiques se calculent par rapport à deux cadres énonciatifs bien distincts : le premier est celui du journaliste, le second celui de l'énonciateur cité ( $c f$. à ce propos Komur 2003a : 132-145 et $2004 \mathrm{~b}: 70$ ). A juste titre, Komur remarque à ce propos que l'ajustement déictique est souvent bloqué par le possessif «nous » (et sa série : «nos, notre »). En fait, dans un discours reformulant, l'équivalent possible de ce déictique n'a pas toujours le même sens que la forme dans le discours d'origine. Le rapporteur journaliste le laisse donc intact. Ainsi, en (8-10), les transpositions automatiques relevant de la reformulation-traduction fabriquent soit une inadéquation de sens, ou directement un contresens par rapport à l'original :

(8) Interrogé par CNN, l'ancien président Bill Clinton a estimé que «sur le plan militaire, nous pouvons battre Saddam Hussein facilement et rapidement ». (Le Monde, vendredi; 14 février $03 / 2) \rightarrow$ ? Interrogé par CNN, l'ancien président Bill Clinton a estimé que «sur le plan militaire, ils pouvaient battre Saddam Hussein facilement et rapidement. »

(9) Il s'agit aujourd'hui, aff,rment Berlin et Paris, d'expérimenter «une union plus étroite entre nos citoyens, nos sociétés et nos institutions ». (Le Monde, jeudi, 23 février $03 / 2) \rightarrow$ Il s'agit aujourd'hui, affirment Berlin et Paris, d'expérimenter « une union plus étroite entre leurs citoyens, leurs sociétés et leurs institutions ».

(10) La veille, dans un entretien publié dans l'hebdomadaire allemand Die Zeit, Luc Ferry, le ministre de l'éducation, proposait d'apprendre l'anglais à l'école primaire et deux autres langues dans le secondaire, "car il serait terrifiant de ne parler entre nous, Allemands et Français, qu'en anglais, et en plus, dans un mauvais anglais». (Le Monde, jeudi, 23 janvier 03/3) $\rightarrow$ La veille, dans un entretien publié dans l'hebdomadaire allemand Die Zeit, Luc Ferry, le ministre de l'éducation, proposait d'apprendre l'anglais à l'école primaire et deux autres langues dans le secondaire, " car il serait terrifiant de ne parler entre eux. Allemands et Français, qu'en anglais, et en plus, dans un mauvais anglais ».

La référence de «ils » dans la partie reformulée est ambiguë : elle peut être prise soit au sens inclusif, soit au sens exclusif ${ }^{14}$ alors que l'emploi de "nous » lève toute ambiguïté (pour plus de détails, $c f$. Komur 2003a : 138-141). Apparemment «déviants » au premier abord, les emplois des îlots textuels montrent une face plus réglée.

Pour notre part, nous aimerions attirer l'attention sur un aspect particulier de la combinatoire libre entre le DC revêtant la forme de l'incise et la Cit que nous avons dénommée mixte. Comme nous l'avons déjà signalé supra, la mixité est d'habitude décrite comme intrusion d'îlots textuels dans un segment reformulé ( $c f$. à ce propos Authier-Revuz 1978: 73-74, Rosier 1999: 233-237). Les expressions guillemetées soit s'adaptent au cadre énonciatif du rapporteur (emploi considéré comme régulier en modalisation autonymique), soit gardent leur propre cadre (cas de l'hybridation des DR).

A nos yeux, l'insertion est possible en sens inverse. Dans le contexte du DR avec incise, on aurait parfois affaire à l'intrusion de segments reformulés dans les reproductions.

Ainsi, les Cit reproductions mixtes consisteraient à intégrer des Cit reformulations dans la Cit reproduction. Leurs déictiques sont ajustés au cadre énonciatif de l'énonciateur cité et non pas à celui du rapporteur et on ne peut pas parler, à cette occasion, d'une hybridation quelconque. Dans ce cas, le rapporteur journaliste rend compte du discours autre en reproduisant la matérialité des propos d'origine tout en y mêlant des reformulés, comme dans les exemples suivants : 
(11) Pour le moment, "il est clair, pour moi, qu'il ne désarme pas », a répété $M$. Bush. ( Le Monde, jeudi, 23 janvier 03/4)

(12) « Nous voulons croire » qu'il est «possible » d'adopter une position commune contre une " action unilatérale » à l'encontre de l'Irak lors de la réunion des ministres des affaires étrangères de l'UE prévue lundi et mardi, a déclaré M. de Villepin lors d'un point de presse (...) (Le Monde , jeudi, 23 janvier 03/4)

En effet, nous avons pu voir que dans les contextes des incises, prédestinées à accueillir des Cit reproductions (nous pensons à des DD canoniques avec incise), la mixité montre encore une facette de plus : elle se figure comme une insertion de reformulé dans du reproduit et neutralise, par là, toute hybridation. A ce qu'il nous semble, les séquences (11-12) peuvent s'interpréter en termes de DD «pseudo-textuel » décrit encore en 1978 par Authier: le segment Cit contient les reproductions en les parsemant de reformulés (1978: 75-76). Le «pseudo-textuel» mêle les caractéristiques du «vrai» direct (au sens de la reproduction fidèle du discours d'origine) avec les indices de reformulation. La Cit mixte contenue dans ces séquences peut être considérée comme une reproduction «à peu près » des paroles d'origine. Seulement, le discours direct pseudo-textuel est, pour Authier (1978: 76), marqué surtout par des éléments spécifiques du DC, tels que : « en substance, en bref, en clair, en gros... etc. » et relève toujours du résumé. Dans nos exemples, le caractère approximatif du rapport de paroles relève du caractère mixte du segment Citation . De la sorte, le rapporteur peut « recréer une parole à la place de mots qu'il n'a pu retenir "par coeur" » (cf. Authier 1978: 76), ou arranger « un peu » des propos qu'il croit répétitifs ou lassants.

\section{En guise de conclusion}

L'îlot textuel ne pénètre jamais sur un terrain énonciatif constant, prédéfini et stable. Tout au contraire, son intervention dans différents domaines énonciatifs entraîne des enjeux inattendus. Il arrive qu'il vienne s'intégrer au DC pour produire des séquences complexes du DR. Lorsqu'il pénètre dans la Cit reformulée en combinatoire contrainte, elle devient Cit reformulation mixte. A côté de l'incise en combinatoire libre, les segments guillemetés accueillent les reformulations pour donner comme résultat des Cit reproductions mixtes. En combinatoire contrainte, le cadre énonciatif de la Cit par rapport au DC peut être soit transposé, soit non transposé ${ }^{15}$. Seul le second cas semble présenter parfois certains indices d'hybridation. En fait, la tâche du rapporteur ne consiste pas à s'adapter aux modèles canoniques du rapport de la parole d'autrui. Il s'adapte plutôt à la réalité crue et aux besoins du contrat de communication. La reproduction et la reformulation ont, en discours, un caractère graduel et évaluatif. Ce n'est pas au grammairien de décider des propriétés (littéral/reformulé) des segments textuels. C'est le rapporteur qui en décide.

\section{Références bibliographiques}

Authier, J. (1978). «Les formes du discours rapporté. Remarques syntaxiques et sémantiques à partir des traitements proposés ». DRLAV, 17, 1-87.

Authier-Revuz, J. (1988). « Non-coïncidences énonciatives dans la production sens ». LINX, 19, 25-28.

Authier-Revuz, J. (1992). « Repères dans le champ du discours rapporté ». L’information grammaticale, 55, 38-42.

Authier-Revuz, J. (1993). «Repères dans le champ du discours rapporté (suite)». L'information grammaticale, 56, $10-15$.

Authier-Revuz, J. (1995) . Ces mots qui ne vont pas de soi. Boucles réflexives et non-coïncidences du dire. Paris : Larousse. 
Authier-Revuz, J. (1996). «Remarques sur la catégorie de l'îlot textuel ». Cahiers du français contemporain, 3, 91115.

Authier-Revuz, J. (1997). «Modalisation autonymique et discours autre: quelques remarques ». Modèles linguistiques, tome XVIII, fascicule 1, 33-51.

Authier-Revuz, J. (1998). « Le guillemet, un signe de "langue écrite" à part entière » dans : J.-M.Defays, L. Rosier \& F. Tilkin (éds), A qui appartient la ponctuation? Louvain-la-Neuve : De Boeck-Duculot, 373-388.

Authier, J. (2004). «La Représentation du discours autre : un champ multiplement hétérogène » dans : J. M. Lopez Muñoz, S. Marnette et L. Rosier (éds), Le discours rapporté dans tous ses états, L'Harmattan, Paris, 35-53.

Bally, Ch. (1912). "Le style indirect libre en français moderne ». Germanisch-Romanische Monatsschrift, 4, 549$556,597-606$.

Bally, Ch. (1914). «Figures de pensée et formes linguistiques ». Germanisch-Romanische Monatsschrift, 6, 405$422,456-470$.

Bakhtine, M. (1977). Le marxisme et la philosophie du langage, traduit du russe par M. Yaguello. Paris : Minuit. Première édition russe publiée sous la signature v.n. Volochinov (1929), Marksizm i filozofija jazyka, Leningrad.

Biardzka, E. (2009). Les échos du «Monde». Pratiques du discours rapporté dans un journal de la presse écrite. Wrocław : Wydawnictwo Uniwersytetu Wrocławskiego.

Durrer S. (1999). Le Dialogue dans le roman. Paris : Nathan.

Gaulmyn de, M.-M. (1983). Les verbes de communication dans la structuration du discours : essai sur la réflexivité du langage - Récits d'enfant et échanges entre enfants et adultes. Thèse d'Etat, Université de Lyon/Université de Paris VIII.

Genette, G. (1972). Figures III. Paris : Seuil.

Greilsamer,L.(réd.) (2004). Le style du « Monde ». Paris : Société éditrice du Monde.

Jakubowska-Cichoń, J. (2010). Mowa przytaczana w narracjach Marguerite Duras. Kraków : Wydawnictwo «Universitas », (à paraître)

Komur, G. (2003a). «Quelques réflexions autour des formes hybrides dans la presse française contemporaine ». Roczniki Humanistyczne, tom LI, 131-145.

Komur, G.(2003b). La représentation du dire dans la presse française contemporaine. Etude qualitative et quantitative de quelques formes de discours rapporté. Thèse du $3^{\mathrm{e}}$ cycle, Université de Paris VIII.

Komur, G. (2004a). «L'îlot textuel et la prise de distance par le locuteur dans le genre journalistique ». dans : J. .Lopez- Muñoz, S. Marnette et L. Rosier (éds), Le discours rapporté dans tous ses états, L'Harmattan, Paris, $54-63$.

Komur, G. (2004b). «Les modes du discours rapporté dans la presse et leurs enjeux polyphoniques ». Pratiques, 123-124, décembre, 57-74.

Labuda, A. W. (1972). «Citation, commentaire et autocommentaire du narrateur. Remarques sur la poétique du texte écrit ». Zagadnienia Rodzajów Literackich, XV (29), 37-51.

Maingueneau, D. (1987). Nouvelles tendances en analyse du discours. Paris : Hachette.

Maingueneau, D. (1994). L'énonciation en linguistique française. Paris : Hachette.

Marczak, E. (2010). Didaskalia narracyjne $w$ « Le Rouge et le Noir » Stendhala. thèse de doctorat non publiée sous la direction de A. W. Labuda, Uniwersytet Wrocławski.

Prince, G. (1978). « Le discours attributif et le récit ». Poétique, 35, 305-313. 
Rosier, L. (1999). Le discours rapporté. Histoire, théorie, pratiques.Paris, Bruxelles : Duculot.

Tuomarlà, U. (2000). La citation mode d'emploi. Sur le fonctionnement discursif du discours rapporté direct. Academia Scientarium Fennica, Ser. Humaniora, tom. 308, Saarijärvi, Finland.

Wilmet, M. (1998). Grammaire critique du français, II ${ }^{\text {ème }}$ édition, revue et corrigée, Paris-Bruxelles : Hachette / Duculot.

\footnotetext{
${ }^{1}$ Date de la traduction française.

2 Parmi les formes simples, elle range quatre cas: le discours direct (avec incise, avec ou sans verbe/ syntagme introducteur), le discours indirect, le discours narrativisé (qu'elle définit comme simple mention de la parole, par exemple sous la forme d'un syntagme nominal ou d'un infinitif qui sont objet d'un verbe locutoire et le discours indirect libre (1983 : 231 Remarquons tout de suite que l'un des emplois « simples » du DR énumérés par Gaulmyn, le discours narrativisé (DN) a été pour la première fois décrit, dans un contexte de recherches littéraires et surtout narratologiques, en premier lieu par Genette (1972: 190-191)
}

${ }^{3}$ Ainsi, nous obtenons : le discours indirect avec îlot textuel inférieur à un syntagme, le discours indirect avec îlot textuel égal ou supérieur à un syntagme, le discours indirect où le « que » est suivi immédiatement des guillemets du $\mathrm{DD}$, le discours narrativisé comportant un îlot textuel égal ou supérieur à un syntagme, le discours narrativisé avec au moins un syntagme entre guillemets, le discours indirect libre comportant un îlot textuel entre guillemets inférieur à un syntagme, et le discours indirect libre comportant un îlot textuel entre guillemets égal ou inférieur à un syntagme.

${ }^{4}$ Remarquons que Charles Bally $(1912,1914)$ et Gérard Genette (1972) identifient le style/discours rapporté uniquement au segment textuel qui représente les paroles citées.

${ }^{5}$ En fait, il suffit de lire un petit bout de texte pour s'apercevoir que les paroles citées sont escortés par des fragments de textes qui ne sont pas eux-mêmes des citations, et qui précisent le contexte de production, la manière dont il faut comprendre les paroles évoquées. $C f$. à ce propos Aleksander Wit Labuda (1972) qui parle de commentaire du narrateur, Gérald Prince (1976) et Sylvie Durrer (1999) qui évoquent le discours attributif, ou Dominique Maingueneau (1994) et Laurence Rosier (1999) qui emploient le terme discours citant. C'est par analogie au théâtre (et au spectacle de paroles de Ducrot) que les chercheurs de l'Université de Wrocław utilisent souvent le terme didascalies proposé par Aleksander Wit Labuda (Elżbieta Biardzka 2009, Joanna Jakubowska-Cichoń 2010 [à paraître], Ewelina Marczak 2010 [ thèse de doctorat non publiée]).

${ }^{6}$ Les majuscules ne sont pas utilisées sans raison : nous prenons les deux termes au sens générique, recouvrant, dans les cas de DC, plusieurs formes grammaticales et textuelles, dans le cas de la Cit, tous les modes de la représentation des paroles citées (aussi bien reproduites « fidèlement» que « reformulées ». La relation syntaxique entre la Cit et le DC dépend de la forme grammaticale que prend ce dernier. Le DC peut prendre plusieurs formes syntaxiques, allant de la simple phrase introductive, passant par la phrase complète, des séquences sans verbe, des syntagmes spécifiques spécialisés dans le rapport de la parole («selon A »), jusqu'à la séquence de phrases (segment textuel compact) ou des formes « originales », difficilement classifiables. En général, les relations entre la DC et la Cit se définissent par deux cas de figure. Soit le locuteur rapporte les paroles de différentes personnes sans les assimiler à la syntaxe de son propre énoncé, soit il les intègre dans son discours conformément aux règles de la syntaxe. Dans le premier cas, nous avons affaire à une combinatoire libre, dans le second cas, nous affrontons une combinatoire contrainte. Dans la combinatoire libre, le DC et la Cit sont syntaxiquement (et typographiquement) séparées, comme dans les textes dramatiques. La séquence du DR se caractérise par une rupture syntaxique: la DC est en quelque sorte «ajouté » à la Cit qui ne semble pas limitée par les formes linguistiques que peut revêtir cette première. La combinatoire libre va au delà des limites de la phrase. Le rapporteur est libre de choisir l'emplacement des DC : leur position par rapport aux Cit n'est pas syntaxiquement contrainte. Pour décrire ce type de fonctionnement du DR, on peut parler de DC transphrastiques. Dans la combinatoire contrainte, le DC et la Cit sont syntaxiquement liés. La séquence du DR qui résulte de la combinatoire contrainte est homogène du point de vue sémiotique et syntaxique, mais elle est hybride du point de vue énonciatif: elle abrite des contenus provenant de deux locuteurs distincts. Ainsi, c'est seulement le segment DC qui contient les propos propres au rapporteur. Dans le segment Cit, ce même rapporteur rapporte un contenu (un signifié) qui ne vient pas de lui : les paroles des énonciateurs cités sont reformulées dans le langage propre au rapporteur ( qui donne à l'énoncé d'origine un nouveau signifiant approprié à 
sa situation d'énonciation). La jonction des DC et des Cit se fait à l'intérieur de la phrase qui est grammaticalement finie et autonome. Le DC est alors intra-phrastique parce qu'il est syntaxiquement soudé à la Cit et non pas « ajouté », comme dans la combinatoire libre.

7 Soulignons que le DR est en fait un bout de texte bipartite. Un bout de texte possède toujours un début et une fin. A nos yeux, sur le plan théorique et empirique, la question de la délimitation des segments DC et Cit (externe et interne) s'avère particulièrement difficile à résoudre : dans beaucoup de cas leurs limites restent incertaines et pour les identifier il faut se laissent guider par l'intuition. Il nous semble pourtant qu'il est possible de recourir à un critère particulièrement valable, celui de l'examen du réseau diaphorique (et éventuellement présuppositionnel et inférentiel) qui se tisse entre les DC et les Cit et qui montre l'homogénéité référentielle des deux segments. Ceci est évidemment le sujet pour un travail à part.

${ }^{8}$ Ainsi, nous ne partageons guère le point de vue de Prince qui estime, à propos du discours attributif (équivalent de ce que nous appelons ici le DC), «qu'on peut très facilement l'isoler» de l'« univers narratif» (1978:313), ni celui de Genette qui voit une limite claire et nette entre le « récit de paroles et le récit d'événements » (1972: 186). Tout au contraire, les limites entre les DC et les séquences du texte appartenant au reste du récit (qui n'est pas le DR) ne nous semblent pas faciles à définir. Le faisceau de critères qui pourraient permettre d'isoler les Did embrasse leur thématique et leurs propriétés de «combinatoire» sémantique et formelle (syntaxique et compositionnelle) avec le récit « muet» (qui ne rapporte pas de paroles).

9 Les exemples cités dans ce travail proviennent du journal Le Monde. Le corpus analysé, décrit dans un travail à part (Biardzka 2009) est le suivant : vendredi, 27 décembre 02; jeudi, 2 janvier 03; vendredi, 3 janvier 03; mardi, 7 janvier 03; mercredi, 8 janvier 03; jeudi, 9 janvier 03; vendredi, 10 janvier 03; dimanche, 12/lundi, 13 janvier 03 ;mardi, 14 janvier 03 ; mercredi, 15 janvier 03 ; jeudi, 16 janvier 03 ; vendredi, 17 janvier 03 ; samedi, 18 janvier 03 ;dimanche, 19/lundi, 20 janvier 03 ; jeudi, 23 janvier 03 ; samedi 25 janvier 03 ; dimanche, 26/lundi 27 janvier 03 ; mardi, 28 janvier 03 ; mercredi, 29 janvier 03 ; jeudi, 6 févier 03 ; samedi, 8 février 03 ; mardi, 11 février 03 ; mercredi, 12 février 03 ; vendredi, 14 février 03 ; dimanche, 16/lundi, 17 février 03 ; vendredi, 21 février 03 ; jeudi 27 février 03 ; mercredi, 5 mars 03 ; vendredi, 7 mars 03 .

10 En fait, la modalisation autonymique, décrite globalement par Authier-Revuz (1993: 14, $2004: 47-49)$ est un phénomène discursif très large. Ainsi, les îlots textuels ne s'insèrent pas seulement dans le DR : ils peuvent s'intégrer dans n'importe quel contexte et il ne manifestera par là, certes, aucun trait de " mixité ».

${ }^{11}$ Les séquences du DR peuvent être répertoriées d'abord selon quatre positions principales que peut prendre le DC par rapport à la Cit : DC antéposé (DC+Cit), DC postposé $(\mathrm{Cit}+\mathrm{DC}), \mathrm{DC}$ intercalé dans la Cit (Cit $+\mathrm{DC}+\mathrm{Cit})$ et DC encadrant la Cit $(\mathrm{DC}+\mathrm{Cit}+\mathrm{DC})$. Puisque ces quatre types principaux de positionnement syntaxique des DC (et, par là, des Cit car nous sommes dans une combinatoire) peuvent se multiplier, on peut prévoir dans une tel inventaire un second paragraphe, consacré aux séquences encore plus développées du DR. (cf. Biardzka 2009:235$305)$.

${ }^{12}$ Pour nous, la dénomination de « mixte » est moins commode car nous l'avons déjà appliquée aux emplois des Cit.

${ }^{13}$ A propos de tels emplois, le guide pour les journalistes du Monde écrit ceci: «Proscrire des formulations telles que : Le premier ministre affirme que " nous avons pris la décision... ". " (Greilsamer 2004 : 17).

${ }^{14}$ Elle est alors en contresens flagrant par rapport au discours d'origine.

${ }^{15}$ Abstraction faite dans ce travail des cas connus de neutralisation. 